astro-ph/0504656

\title{
CMB Anisotropy of Spherical Spaces
}

\author{
Ralf Aurich ${ }^{1}$, Sven Lustig ${ }^{1}$, Frank Steiner ${ }^{1,2}$ \\ ${ }^{1}$ Abteilung Theoretische Physik, Universität Ulm, \\ Albert-Einstein-Allee 11, D-89069 Ulm, Germany \\ ${ }^{2}$ Theory Division, Physics Department, \\ CERN, CH-1211 Geneva 23, Switzerland
}

\begin{abstract}
The first-year WMAP data taken at their face value hint that the Universe might be slightly positively curved and therefore necessarily finite, since all spherical (Clifford-Klein) space forms $\mathcal{M}^{3}=\mathcal{S}^{3} / \Gamma$, given by the quotient of $\mathcal{S}^{3}$ by a group $\Gamma$ of covering transformations, possess this property. We examine the anisotropy of the cosmic microwave background $(\mathrm{CMB})$ for all typical groups $\Gamma$ corresponding to homogeneous universes. The CMB angular power spectrum and the temperature correlation function are computed for the homogeneous spaces as a function of the total energy density parameter $\Omega_{\text {tot }}$ in the large range $[1.01,1.20]$ and are compared with the WMAP data. We find that out of the infinitely many homogeneous spaces only the three corresponding to the binary dihedral group $T^{\star}$, the binary octahedral group $O^{\star}$, and the binary icosahedral group $I^{\star}$ are in agreement with the WMAP observations. Furthermore, if $\Omega_{\text {tot }}$ is restricted to the interval [1.00,1.04], the space described by $T^{\star}$ is excluded since it requires a value of $\Omega_{\text {tot }}$ which is probably too large being in the range $[1.06,1.07]$. We thus conclude that there remain only the two homogeneous spherical spaces $\mathcal{S}^{3} / O^{\star}$ and $\mathcal{S}^{3} / I^{\star}$ with $\Omega_{\text {tot }}$ of about 1.038 and 1.018 , respectively, as possible topologies for our Universe.

PACS numbers: 98.80.-k, 98.70.Vc, 98.80.Es
\end{abstract}

\section{Introduction}

At present, all data are consistent with, and in fact strongly support, the standard bigbang model in which the time evolution of the Universe is described by the FriedmannLemaittre-Robertson-Walker metric. Accordingly, the Universe possesses the space-time structure $\mathbb{R} \times \mathcal{M}$ where $\mathbb{R}$ describes the "space" of cosmic time, and $\mathcal{M}$ the threedimensional comoving space section of constant curvature $K=+1,0$ and -1 . The Einstein field equations respectively the Friedmann equations for the cosmic scale factor do not fix the curvature a priori. Instead, the curvature parameter $K$ has to be inferred from a determination of the total energy density $\varepsilon_{\text {tot }}$ of the Universe via the relation $(c=1) K=H_{0}^{2} a_{0}^{2}\left(\Omega_{\text {tot }}-1\right), \Omega_{\text {tot }}:=\varepsilon_{\text {tot }} / \varepsilon_{\text {crit }}$, where $\varepsilon_{\text {crit }}:=\frac{3 H_{0}^{2}}{8 \pi G}$ denotes the critical energy density, $a_{0}$ the cosmic scale factor, and $H_{0}$ the Hubble constant (all quantities at the present epoch). Furthermore, it is a mathematical fact, although not always appreciated (see, however, the remark on early works below), that fixing the curvature 
$K$ does not determine uniquely the global geometry of $\mathcal{M}$, i. e. the topology and thus the shape of the Universe. Only if it is assumed that the Universe is simply-connected, the possible homogeneous 3 -spaces $\mathcal{M}$ of constant curvature $K$ are given by the 3 -sphere $\mathcal{S}^{3}(K=+1)$, Euclidean 3 -space $\mathcal{E}^{3}(K=0)$, or hyperbolic 3 -space $\mathcal{H}^{3}(K=-1)$. In this case, the Universe is finite for positive curvature $\left(\Omega_{\text {tot }}>1\right)$ and infinite for vanishing $\left(\Omega_{\text {tot }}=1\right)$ or negative curvature $\left(\Omega_{\text {tot }}<1\right)$. However, most 3 -spaces $\mathcal{M}$ of constant curvature are multi-connected and are given by the quotient of $\mathcal{S}^{3}, \mathcal{E}^{3}$, or $\mathcal{H}^{3}$ by a group $\Gamma$ of covering transformations, i. e. $\mathcal{M}=\mathcal{S}^{3} / \Gamma, \mathcal{E}^{3} / \Gamma$, or $\mathcal{H}^{3} / \Gamma$. In this case, the Universe is again finite for positive curvature, but can be finite too if it is flat or negatively curved.

Here, we would like to remark that the question whether the space of the Universe is finite and possibly multi-connected has been discussed during the last century by several cosmologists, e.g. by Schwarzschild [1], Einstein [2], Friedmann [3, 4], Lemaître [5], Heckmann and Schücking [6], and Ellis [7], to mention only a few.

The concordance model of cosmology ( $\Lambda$ CDM model) assumes a flat Universe with the topology of $\mathcal{E}^{3}$ with a positive cosmological constant $\Lambda$, i. e. $\Omega_{\Lambda}:=\frac{\Lambda}{3 H_{0}^{2}}=$ $1-\Omega_{\text {mat }}-\Omega_{\text {rad }}$ with $\Omega_{\text {mat }}=\Omega_{\text {bar }}+\Omega_{\mathrm{cdm}}$, where the various $\Omega$-parameters denote the present value of the baryonic (bar), cold dark matter (cdm), matter (mat) and radiation (rad) energy densities in units of $\varepsilon_{\text {crit }}$. Three variants of the concordance model have been presented by the WMAP team [8] providing a good overall fit to the temperature fluctuations $\delta T$ of the cosmic microwave background radiation (CMB) on small and medium scales, but there remains a strange discrepancy at large scales as first observed by COBE [9] and later substantiated by WMAP [10].

The suppression of the CMB anisotropy at large scales respectively low multipoles can be explained if the Universe is finite. Recent analyses concerning the suppression at low multipoles in the WMAP data can be found in 11, 12, 13, 14, 15. As discussed before, a finite Universe is naturally obtained, if the total energy density exceeds the critical value one, i. e. $\Omega_{\text {tot }}>1$. Interestingly enough, the WMAP team reported [10] $\Omega_{\mathrm{tot}}=1.02 \pm 0.02$ together with $\Omega_{\mathrm{bar}}=0.044 \pm 0.004, \Omega_{\mathrm{mat}}=0.27 \pm 0.04$, and $h=0.71_{-0.03}^{+0.04}$ for the present day reduced Hubble constant (the errors give the $1 \sigma$ deviation uncertainties only). Taking at their face value, these parameters hint to a positively curved Universe possessing the geometry of $\mathcal{S}^{3}$ or of one of the spatial space forms $\mathcal{S}^{3} / \Gamma$. (One should keep in mind, however, that the WMAP values depend on certain priors, and, furthermore, include the $1 \sigma$-errors only. Thus it would be too early to conclude that the data definitively exclude a negatively curved Universe. In fact, we have recently shown [12, 13] that the non-compact, but finite hyperbolic Picard universe describes well the CMB anisotropy and the observed suppression of power at large scales.)

In this paper, we present a systematic comparison of the predictions with the CMB anisotropy for universes possessing homogeneous spherical topology. This comparison is made possible for two reasons. First of all, the spherical spaces were classified already by 1932 [16, 17] and thus their mathematical structure is known. (This is in contrast to the case of hyperbolic manifolds which are not yet completely classified; even the manifold 
with the smallest volume is not yet known.) Second, due to an efficient numerical algorithm described in our recent paper [14, we are able to take in the Sachs-Wolfe formula a large number of vibrational modes into account and thus to predict sufficiently many CMB multipoles for the various spherical spaces which in turn allow a detailed comparison with the WMAP data. Since the CMB spectrum depends sensitively on the curvature radius, the comparison is performed as a function of $\Omega_{\text {tot }}$ in the large interval $[1.01,1.20]$ in order to determine for a given spherical space form the bestfitting value of the total energy density - under the condition, of course, that the space under consideration is able to describe the data at all.

Recently, Luminet et al. 11 proposed the Poincaré dodecahedron, which is one of the well-known spherical space forms (see section 2 for details), as a model for the geometry of the Universe. In their preliminary study involving only the three lowest multipoles $(l=2,3,4)$ they found, indeed, for $\Omega_{\text {tot }}=1.013$ a strong suppression of the CMB power at $l=2$ and a weak suppression at $l=3$ in agreement with the WMAP data. However, in [11] only the first three vibrational modes of the dodecahedral space with wave number $\beta=13,21$ and 25 (comprising in total 59 eigenfunctions) have been used, and there thus remained the question about how this extremely low wave number cut-off affects the predictions of the multipoles, since experience shows that increasing the cut-off usually enhances the integrated Sachs-Wolfe and Doppler contributions.

In our recent paper [14] we presented a thorough discussion of the CMB anisotropy for the dodecahedral space topology using the first 10521 eigenfunctions corresponding to the large wave number cut-off $\beta=155$. The contributions of higher wave numbers up to $\beta=1501$ were taken into account with respect to their mean behaviour. Taking within the tight-coupling approximation not only the ordinary, but also the integrated SachsWolfe and also the Doppler contribution into account, we were able to predict sufficiently many multipole moments such that a detailed comparison of the dodecahedral space model with the WMAP data could be performed. We found that the temperature correlation function for the dodecahedral universe possesses very weak correlations at large scales in nice agreement with the WMAP data for $\Omega_{\text {tot }}$ in the range $1.016 \ldots 1.020$. There thus arises the interesting question whether the dodecahedral space is the only spherical space form able to describe the CMB data. In [15] the CMB anisotropy is also studied for the dodecahedron, the binary octahedral group, and the binary tetrahedral group.

The main result of the present paper is that while almost all homogeneous spherical spaces have to be excluded as possible geometries for the Universe, there is one particular space form, defined by the binary octahedral group, which agrees for $\Omega_{\text {tot }} \simeq 1.038$ with the WMAP data even better than the dodecahedron. We observe that the bestfitting values obtained for $\Omega_{\text {tot }}$ are different for the binary octahedral space and the dodecahedron, but both values lie well within the $1 \sigma$-band determined by WMAP. It remains to be seen whether future data will enable us to definitely eliminate one of the two space forms in favour of the other as describing the true topology of the Universe.

Our paper is organised as follows. In section 2, we summarize the main properties 
of the existing homogeneous spherical space forms and of their vibrational modes. Our main results are presented in section 3 which contains a detailed comparison with the WMAP data of the CMB angular power spectrum for the various types of spherical spaces. In addition to the power spectrum, we study also the so-called $S(\rho)$ statistic 10. which measures the suppression at large angular scales directly in terms of the temperature correlation function. Section 4 contains our conclusions.

\section{The spherical space forms and their vibrational modes}

In section 2 of [14] we have already described the three-dimensional spaces $\mathcal{M}$ of constant positive curvature $K=1$, and therefore we refer the reader to this paper for details. The spherical spaces were classified by 1932 [16, 17] and are given by the quotient $\mathcal{M}=\mathcal{S}^{3} / \Gamma$ of the three-sphere $\mathcal{S}^{3}$ under the action of a discrete fixed-point free subgroup $\Gamma \subset \mathrm{SO}(4)$ of the isometries of $\mathcal{S}^{3}$. All these manifolds are compact possessing the volume $V\left(\mathcal{S}^{3} / \Gamma\right)=V\left(\mathcal{S}^{3}\right) / N$, where $N$ is the order of the group $\Gamma$, and are, apart from the universal covering space $\mathcal{S}^{3}$ with volume $V\left(\mathcal{S}^{3}\right)=2 \pi^{2}$, multi-connected. To define the discrete fixed-point free subgroups $\Gamma \subset \mathrm{SO}(4)$ of isometries of $\mathcal{S}^{3}$, one makes use of the fact that the unit 3 -sphere $\mathcal{S}^{3}$ can be identified with the multiplicative group of unit quaternions $\{q\}$. The latter are defined by $q:=w+x \mathbf{i}+y \mathbf{j}+z \mathbf{i j},(w, x, y, z) \in \mathbb{R}^{4}$, having unit norm, $|q|^{2}=w^{2}+x^{2}+y^{2}+z^{2}=1$. Here, the 4 basic quaternions $\{1, \mathrm{i}, \mathrm{j}, \mathrm{ij}\}$ satisfy the multiplication rules $\mathrm{i}^{2}=\mathrm{j}^{2}=-1$ and $\mathrm{ij}=-\mathrm{ji}$ plus the property that $\mathrm{i}$ and $\mathrm{j}$ commute with every real number. The distance $d\left(q_{1}, q_{2}\right)$ between two points $q_{1}$ and $q_{2}$ on $\mathcal{S}^{3}$ is given by $\cos d\left(q_{1}, q_{2}\right)=w_{1} w_{2}+x_{1} x_{2}+y_{1} y_{2}+z_{1} z_{2}$.

The group $\mathrm{SO}(4)$ is isomorphic to $\mathcal{S}^{3} \times \mathcal{S}^{3} /\{ \pm(1,1)\}$, the two factors corresponding to the left and right group actions. In this paper, we are only interested in homogeneous manifolds $\mathcal{M}=\mathcal{S}^{3} / \Gamma$, in which case the group $\Gamma$ contains only right-handed Clifford translations $\gamma \in \Gamma$ that act on an arbitrary unit quaternion $q \in \mathcal{S}^{3}$ by left-multiplication, $q \rightarrow \gamma q$, and translate all points $q_{1}, q_{2} \in \mathcal{S}^{3}$ by the same distance $\chi$, i. e. $d\left(q_{1}, \gamma q_{1}\right)=$ $d\left(q_{2}, \gamma q_{2}\right)=\chi$. The right-handed Clifford translations act as right-handed cork screw fixed-point free rotations of $\mathcal{S}^{3}$. The following groups lead to homogeneous manifolds $\mathcal{M}=\mathcal{S}^{3} / \Gamma$ [16, 17, 18, 19]:

- The cyclic groups $Z_{m}$ of order $m(m \geq 1)$.

- The binary dihedral groups $D_{4 m}^{\star}$ of order $4 m(m \geq 2)$.

- The binary tetrahedral group $T^{\star}$ of order 24 .

- The binary octahedral group $O^{\star}$ of order 48 .

- The binary icosahedral group $I^{\star}$ of order 120 .

In table 1 we give the right-handed Clifford translations which generate the above groups $\Gamma$.

The vibrations on the homogeneous spherical spaces $\mathcal{M}=\mathcal{S}^{3} / \Gamma$ are determined by the regular solutions of the Helmholtz equation

$$
\left(\Delta+E_{\beta}^{\mathcal{M}}\right) \psi_{\beta}^{\mathcal{M}, i}(q)=0 \quad, \quad q \in \mathcal{M},
$$




\begin{tabular}{|c|c|c|}
\hline$\Gamma$ & $\gamma_{1}$ & $\gamma_{2}$ \\
\hline$Z_{m}$ & $\cos \left(\frac{2 \pi}{m}\right)+\mathrm{i} \sin \left(\frac{2 \pi}{m}\right)$ & - \\
\hline$D_{4 m}^{\star}$ & $\cos \left(\frac{2 \pi}{m}\right)+\mathrm{ij} \sin \left(\frac{2 \pi}{m}\right)$ & $\mathrm{i}$ \\
\hline$T^{\star}$ & $\mathrm{j}$ & $\frac{1}{2}+\frac{1}{2} \mathrm{i}+\frac{1}{2} \mathrm{j}+\frac{1}{2} \mathrm{ij}$ \\
\hline$O^{\star}$ & $\frac{1}{\sqrt{2}}+\frac{1}{\sqrt{2}} \mathrm{i}$ & $\frac{1}{2}+\frac{1}{2} \mathrm{i}+\frac{1}{2} \mathrm{j}+\frac{1}{2} \mathrm{ij}$ \\
\hline$I^{\star}$ & $\mathrm{j}$ & $\frac{\sigma}{2}+\frac{1}{2 \sigma} \mathrm{i}+\frac{1}{2} \mathrm{j}$ \\
\hline
\end{tabular}

Table 1. The generators $\gamma_{1}$ and $\gamma_{2}$ for the groups $\Gamma(\sigma=(1+\sqrt{5}) / 2)$.

satisfying the fundamental periodicity conditions

$$
\psi_{\beta}^{\mathcal{M}, i}\left(\gamma_{k} q\right)=\psi_{\beta}^{\mathcal{M}, i}(q) \quad, \quad \forall q \in \mathcal{M}, \forall \gamma_{k} \in \Gamma .
$$

Here $\Delta$ denotes the Laplace-Beltrami operator on $\mathcal{S}^{3}$. The eigenfunctions on $\mathcal{M}$ satisfy the orthonormality relation

$$
\int_{\mathcal{M}} d \mu \psi_{\beta}^{\mathcal{M}, i}(q) \psi_{\beta^{\prime}}^{\mathcal{M}, i^{\prime}}(q)=\frac{1}{N} \int_{\mathcal{S}^{3}} d \mu \psi_{\beta}^{\mathcal{M}, i}(q) \psi_{\beta^{\prime}}^{\mathcal{M}, i^{\prime}}(q)=\delta_{\beta \beta^{\prime}} \delta i i^{\prime} .
$$

The spectrum on $\mathcal{M}$ is discrete, and the eigenvalues can be expressed in terms of the wave number $\beta \in \mathbb{N}$ as $E_{\beta}=\beta^{2}-1$ and are independent of the degeneracy index $i=1, \ldots, r^{\mathcal{M}}(\beta)$, where $r^{\mathcal{M}}(\beta)$ denotes the multiplicity of the mode $\beta$. It should be noted that for a given manifold $\mathcal{M}$ the wave numbers $\beta$ do not take all values in $\mathbb{N}$. The allowed $\beta$ values together with their multiplicities $r^{\mathcal{M}}(\beta)$ are explicitly known [20, 21], see Table 2 .

The eigenfunctions $\psi_{\beta}^{\mathcal{M}, i}(q)$ on $\mathcal{M}$ can be expanded into the eigenfunctions $\psi_{\beta l m}^{\mathcal{S}^{3}}(q)$ on $\mathcal{S}^{3}$

$$
\psi_{\beta}^{\mathcal{M}, i}(q)=\sum_{l=0}^{\beta-1} \sum_{m=-l}^{l} \xi_{\beta l m}^{i}(\mathcal{M}) \psi_{\beta l m}^{\mathcal{S}^{3}}(q) .
$$

Since the eigenfunctions on $\mathcal{S}^{3}$ are explicitly known [22, 23, 24, 25, 26, 27], it remains to determine the expansion coefficients $\xi_{\beta l m}^{i}(\mathcal{M})$ which satisfy as a consequence of eq. (3) the normalization condition

$$
\sum_{l=0}^{\beta-1} \sum_{m=-l}^{l}\left(\xi_{\beta l m}^{i}(\mathcal{M})\right)^{2}=N
$$

In [14] we have described our numerical algorithm to compute the coefficients $\xi_{\beta l m}^{i}(\mathcal{M})$. It uses a collocation method by imposing the periodicity condition (2). Using this method, we have computed in [14 the expansion coefficients for $\Gamma=I^{\star}$, i. e. for the Poincaré dodecahedral space $\mathcal{D}=\mathcal{S}^{3} / I^{\star}$, for $\beta \leq 155$ comprising the first 10521 eigenfunctions. In addition, we have computed in [14] the coefficients $\xi_{\beta l m}^{i}(\mathcal{M})$ for $\beta \leq 33$ for some cyclic groups, some binary dihedral groups, the binary tetrahedral group, and the binary octahedral group. Based on these numerical results, we stated for homogeneous space forms the following relation as a conjecture $(0 \leq l \leq \beta-1)$

$$
\frac{1}{2 l+1} \sum_{m=-l}^{l} \sum_{i=1}^{r^{\mathcal{M}}(\beta)}\left(\xi_{\beta l m}^{i}(\mathcal{M})\right)^{2}=N \frac{r^{\mathcal{M}}(\beta)}{r^{\mathcal{S}^{3}}(\beta)},
$$


where $r^{\mathcal{S}^{3}}(\beta)=\beta^{2}$ denotes the multiplicity of the vibrational modes on $\mathcal{S}^{3}$. The relation (6) has been found to hold within a numerical accuracy of 13 digits. However, for the inhomogeneous lens spaces [28] $\mathrm{L}(12,5)$ and $\mathrm{L}(72,17)$ we have found that the relation (6) does not hold. We thus concluded in [14] that the relation (6) is only valid for homogeneous 3-spaces. Recently, Gundermann [15] provided a proof of our conjecture (6).

In the next section, we shall use the eigenfunctions (44) to calculate CMB sky maps and the variance of the CMB anisotropy and relation (6) to calculate the mean value of the CMB anisotropy for a variety of spherical spaces.

\section{The angular power spectrum $\delta T_{l}^{2}$ and the correlation function $C(\vartheta)$ for spherical spaces}

The relative temperature fluctuations $\frac{\delta T(\hat{n})}{T}$ of the CMB are caused by several effects which we shall compute within the tight-coupling approximation along the lines described in detail in Section 2 of [12. The dominant contribution at large scales is given by the ordinary Sachs-Wolfe (SW) effect which is a combination of the gravitational potential $\Phi(\eta, \tau, \theta, \phi)$ at the surface of last scattering (SLS), and the intrinsic temperature fluctuation $\frac{1}{4} \delta_{\gamma}(\eta, \tau, \theta, \phi)$ due to the imposed entropic initial conditions, where $\delta_{\gamma}$ denotes the relative perturbation in the radiation component. (Here $\eta$ denotes the conformal time and $(\tau(\eta), \theta, \phi)$ the spherical coordinates of the photon path in the direction $\hat{n}=\hat{n}(\theta, \phi)$, where we assume that the observer is at the origin of the coordinate system, i. e. at $\left(\tau_{\text {obs }}, \theta_{\text {obs }}, \phi_{\text {obs }}\right)=(0,0,0)$.)

The gravitational potential $\Phi$ is identified with the (scalar) perturbation of the Friedmann-Lemaître-Robertson-Walker metric which for an energy-momentum tensor $T_{\mu \nu}$ with $T_{i j}=0$ for $i \neq j(i, j=1,2,3)$ can in conformal Newtonian gauge be written as 29$]$

$$
d s^{2}=a^{2}(\eta)\left[(1+2 \Phi) d \eta^{2}-(1-2 \Phi)|d \vec{x}|^{2}\right] .
$$

Here $a(\eta)$ denotes the cosmic scale factor as a function of conformal time $\eta$ and $|d \vec{x}|^{2}$ the line element on $\mathcal{S}^{3}$

$$
|d \vec{x}|^{2}=d \tau^{2}+\sin ^{2} \tau\left(d \theta^{2}+\sin ^{2} \theta d \phi^{2}\right)
$$

with $0 \leq \tau \leq \pi, 0 \leq \theta \leq \pi, 0 \leq \phi \leq 2 \pi$.

The ordinary Sachs-Wolfe (SW) contribution to the temperature fluctuation is given by

$$
\frac{\delta T^{\mathrm{SW}}(\hat{n})}{T}=\Phi\left(\eta_{\mathrm{SLS}}, \tau_{\mathrm{SLS}}, \theta, \phi\right)+\frac{1}{4} \delta_{\gamma}\left(\eta_{\mathrm{SLS}}, \tau_{\mathrm{SLS}}, \theta, \phi\right)
$$

with $\tau_{\mathrm{SLS}}:=\eta_{0}-\eta_{\mathrm{SLS}}$, where $\eta_{0}$ and $\eta_{\mathrm{SLS}}$ denote the conformal time at the present epoch and at the time of recombination corresponding to a redshift $z_{\mathrm{SLS}}=1089$, respectively. 
For a given spherical space $\mathcal{M}$, the metric perturbation can be written as an expansion in the eigenfunctions on $\mathcal{M}$

$$
\Phi(\eta, \tau, \theta, \phi)=\sum_{\beta \geq 3}^{\prime} \sum_{i=1}^{r^{\mathcal{M}}(\beta)} \Phi_{\beta}^{i}(\eta) \Psi_{\beta}^{\mathcal{M}, i}(\tau, \theta, \phi)
$$

where in the mode summation only the modes with $\beta \geq 3$ have been taken into account (if they exist), since the wave numbers $\beta=1,2$ correspond to modes which are pure gauge terms [29]. The prime in the summation over the modes $\beta$ indicates that the spectrum of a given manifold $\mathcal{M}$ does not contain all $\beta \in \mathbb{N}$, see Table 2. The functions $\Phi_{\beta}^{i}(\eta)$ determine the time evolution and will be factorized $\Phi_{\beta}^{i}(\eta)=\Phi_{\beta}^{i}(0) g_{\beta}(\eta)$ with $g_{\beta}(0)=1$. The functions $g_{\beta}(\eta)$ do not depend on the degeneracy index $i$, since the associated differential equation depends only on the eigenvalue $E_{\beta}^{\mathcal{M}}$ which is independent of $i$. The initial values $\Phi_{\beta}^{i}(0)$ are the primordial fluctuation amplitudes and are assumed to be Gaussian random variables with zero expectation value and covariance

$$
\left\langle\Phi_{\beta}^{i}(0) \Phi_{\beta^{\prime}}^{i^{\prime}}(0)\right\rangle=\delta_{\beta \beta^{\prime}} \delta_{i i^{\prime}} P_{\Phi}(\beta) .
$$

Here $P_{\Phi}(\beta)$ denotes the primordial power spectrum that determines the weight by which the primordial modes $\beta$ are excited, on average. The average $\langle\ldots\rangle$ in (11) denotes an ensemble average over the primordial perturbations which are supposed to arise from quantum fluctuations, by which the Universe is "created". In the following, we shall assume that the primordial power spectrum is in good approximation described by the scale-invariant Harrison-Zel'dovich spectrum

$$
P_{\Phi}(\beta)=\frac{\alpha}{\beta\left(\beta^{2}-1\right)} .
$$

Here $\alpha$ is a normalization factor which will be determined from the CMB data.

The temperature fluctuations $\delta T(\hat{n})$ of the microwave sky can be expanded into real spherical harmonics $\tilde{Y}_{l m}(\hat{n})$ on $\mathcal{S}^{2}$,

$$
\delta T(\hat{n}):=\sum_{l=2}^{\infty} \sum_{m=-l}^{l} a_{l m} \tilde{Y}_{l m}(\hat{n})
$$

where the monopole and dipole terms, $l=0,1$, are not included in the sum (13). From the real expansion coefficients $a_{l m}$ one forms the multipole moments

$$
C_{l}:=\frac{1}{2 l+1}\left\langle\sum_{m=-l}^{l}\left(a_{l m}\right)^{2}\right\rangle
$$

and the angular power spectrum

$$
\delta T_{l}^{2}:=\frac{l(l+1)}{2 \pi} C_{l}
$$

The average $\langle\ldots\rangle$ in (14) denotes an ensemble average over the primordial perturbations as in eq.(11), respectively an ensemble average over the universal observers. 
Inserting the expansions (10) and (41) into the approximation $\frac{\delta T^{\mathrm{SW}}(\hat{n})}{T} \simeq$ $\frac{1}{3} \Phi\left(\eta_{\mathrm{SLS}}, \tau_{\mathrm{SLS}}, \theta, \phi\right)$ to the Sachs-Wolfe formula (9) and using the explicit expression for the eigenfunctions $\psi_{\beta l m}^{\mathcal{S}^{3}}$ on $\mathcal{S}^{3}$,

$$
\psi_{\beta l m}^{\mathcal{S}^{3}}(\vec{x})=R_{\beta l}(\tau) \tilde{Y}_{l m}(\theta, \phi),
$$

one arrives [14] with the help of relation (6) at the following expression for the ordinary Sachs-Wolfe contribution to the multipole moments for a given spherical space $\mathcal{M}=\mathcal{S}^{3} / \Gamma$

$$
C_{l}^{\mathrm{SW}}(\mathcal{M})=\frac{N}{9} \sum_{\beta>l}^{\prime} \frac{r^{\mathcal{M}}(\beta)}{\beta^{2}} P_{\Phi}(\beta) g_{\beta}^{2}\left(\eta_{\mathrm{SLS}}\right) R_{\beta l}^{2}\left(\tau_{\mathrm{SLS}}\right)
$$

Here $R_{\beta l}(\tau)$ denote the "radial functions" on $\mathcal{S}^{3}$ which can be expressed in terms of Gegenbauer polynomials, see eq.(10) of [14]. The expression (17) shows in a transparent way, why the lowest multipoles are in general suppressed for the multi-connected spherical spaces, the more the more wave numbers $\beta$ are missing in the vibrational spectrum. Let us consider, for example, the quadrupole moment, $l=2$. Then the summation over modes in (17) runs for the simply-connected manifold $\mathcal{S}^{3}$ over all natural numbers with $\beta \geq 3$. In contrast, for the Poincaré dodecahedral space $\mathcal{D}$ there is a large gap between $\beta=3$ and 12 , since the lowest contributing mode occurs only at $\beta=13$, and thus the missing modes lead to a suppression. The suppression on $\mathcal{D}$ gets even stronger, because there exist only the three modes $\beta=21,25$ and 31 in the wave number interval $13<\beta<33$ (see table 2).

Now, we would like to discuss this suppression mechanism in more detail. Table 2 shows that the allowed wave numbers $\beta$ are for all homogeneous spherical spaces given by odd natural numbers, except for the homogeneous lens spaces $\mathcal{S}^{3} / Z_{m}$ with $m$ odd $\geq 1$ for which $\beta$ runs through all natural numbers with $\beta \geq m+1$, in addition to the lowest odd wave numbers between 1 and $m$. While the $\beta$ spectrum consists for the homogeneous lens spaces $Z_{m}$ with $m$ even $\geq 2$ of all odd natural numbers, there are for all other spherical spaces at low wave numbers below a given threshold $\beta_{\text {th }}$ "gaps" in the spectrum. As can be seen from Table 2, the threshold value $\beta_{\text {th }}$ increases if the volume $V(\mathcal{M})$ decreases, i. e. $\beta_{\text {th }}=4[(m+1) / 2]+1,13,25$ and 61 for the spaces belonging to the groups $D_{4 m}^{\star}, T^{\star}, O^{\star}$ and $I^{\star}$, respectively. This demonstrates clearly the important rôle played by the spatial volume, i. e. the topology of the Universe.

In addition to the wave number gaps, there is another important imprint of topology on the multipole moments $C_{l}$ due to the multiplicities $r^{\mathcal{M}}(\beta)$, also given in Table 2, by which the vibrational modes are weighted in the mode sum (17). Again, this effect is significant for the low vibrational modes and thus for the low multipole moments which can be considered as carrying the fingerprints of the topology of the Universe.

On the other hand, for the large multipole moments, $l \gg \beta_{\mathrm{th}}$, the details of the different topologies get washed out due to the identical mean asymptotic behaviour of the multiplicities for all multi-connected spaces $\mathcal{M}$ (except $Z_{m}, m$ odd) given by

$$
r^{\mathcal{M}}(\beta)=\frac{2}{N} \beta^{2}+\ldots=\frac{V(\mathcal{M})}{\pi^{2}} \beta^{2}+\ldots \text { for } \quad \beta \rightarrow \infty
$$




\begin{tabular}{|c|c|c|}
\hline$\Gamma$ & wave number spectrum $\{\beta\}$ of manifold $\mathcal{M}=\mathcal{S}^{3} / \Gamma$ & multiplicity $r^{\mathcal{M}}(\beta)$ \\
\hline$Z_{1}$ & $\mathbb{N}$ & $\beta^{2}$ \\
\hline$Z_{m}, m$ odd $\geq 1$ & $\{1,3,5, \ldots, m\} \cup\{n \mid n \geq m+1\}$ & $\beta \sum_{\beta-1 \equiv 2 l(m) ; 0 \leq l \leq \beta-1} 1$ \\
\hline$Z_{m}, m$ even $\geq 2$ & $2 \mathbb{N}+1$ & $\beta \sum_{\beta-1 \equiv 2 l(m) ; 0 \leq l \leq \beta-1} 1$ \\
\hline$D_{4 m}^{\star}, m \geq 2$ & $\left\{1,5,9, \ldots, 4\left[\frac{m+1}{2}\right]+1\right\} \cup\left\{2 n+1 \mid n \geq 2\left[\frac{m+1}{2}\right]+1\right\}$ & $\beta\left(\left[\frac{\beta-1}{2 m}\right]+1\right)$ for $\beta \in\{4 n+1 \mid n \geq 0\}$ \\
& & $\beta\left[\frac{\beta-1}{2 m}\right]$ for $\beta \in\left\{4 n+3 \mid n \geq\left[\frac{m+1}{2}\right]\right\}$ \\
\hline$T^{\star}$ & $\{1,7,9\} \cup\{2 n+1 \mid n \geq 6\}$ & $\beta\left(2\left[\frac{\beta-1}{6}\right]+\left[\frac{\beta-1}{4}\right]-\frac{\beta-3}{2}\right)$ \\
\hline$O^{\star}$ & $\{1,9,13,17,19,21\} \cup\{2 n+1 \mid n \geq 12\}$ & $\beta\left(\left[\frac{\beta-1}{8}\right]+\left[\frac{\beta-1}{6}\right]+\left[\frac{\beta-1}{4}\right]-\frac{\beta-3}{2}\right)$ \\
\hline$I^{\star}$ & $\{1,13,21,25,31,33,37,41,43,45,49,51,53,55,57\}$ & $\beta\left(\left[\frac{\beta-1}{10}\right]+\left[\frac{\beta-1}{6}\right]+\left[\frac{\beta-1}{4}\right]-\frac{\beta-3}{2}\right)$ \\
& $\cup\{2 n+1, n \geq 30\}$ & \\
\hline
\end{tabular}

Table 2. The eigenvalue spectrum for the groups $\Gamma$.

which leads to the universal formula $\left(l \gg \beta_{\text {th }}\right)$

$$
C_{l}^{\mathrm{SW}}(\mathcal{M}) \simeq \frac{2}{9} \sum_{\beta \text { odd }, \beta>l}^{\infty} P_{\Phi}(\beta) g_{\beta}^{2}\left(\eta_{\mathrm{SLS}}\right) R_{\beta l}^{2}\left(\tau_{\mathrm{SLS}}\right) .
$$

For the homogeneous lens spaces $Z_{m}, m$ odd $\geq 1$, one obtains instead

$$
r^{\mathcal{M}}(\beta)=\frac{1}{N} \beta^{2}+\ldots \quad \text { for } \quad \beta \rightarrow \infty,
$$

and thus from (17) for $l \gg \beta_{\text {th }}$

$$
C_{l}^{\mathrm{SW}}\left(Z_{m}\right) \simeq \frac{1}{9} \sum_{\beta=l+1}^{\infty} P_{\Phi}(\beta) g_{\beta}^{2}\left(\eta_{\mathrm{SLS}}\right) R_{\beta l}^{2}\left(\tau_{\mathrm{SLS}}\right)
$$

which should be numerically not very different from the expression (19) where the additional factor 2 should recompensate for the missing even $\beta$-values in the sum (19). The asymptotic behaviour given in eqs. (18) and (20) is in agreement with Weyl's law, as can be seen as follows $(k \rightarrow \infty)$ :

$$
\begin{aligned}
\mathcal{N}^{\mathcal{M}}(k) & :=\#\{\beta \mid \beta \leq k\}=\sum_{\beta \leq k}^{\prime} r^{\mathcal{M}}(\beta) \\
& =\sum_{\beta \text { odd, }, \beta \leq k}\left(\frac{2}{N} \beta^{2}\right)+\ldots=\frac{V(\mathcal{M})}{6 \pi^{2}} k^{3}+\ldots
\end{aligned}
$$

for $\Gamma \neq Z_{m}, m$ odd $\geq 1$, and

$$
\mathcal{N}^{\mathcal{M}}(k)=\sum_{\beta=1}^{k}\left(\frac{1}{N} \beta^{2}\right)+\ldots=\frac{V(\mathcal{M})}{6 \pi^{2}} k^{3}+\ldots
$$

for $\Gamma=Z_{m}, m$ odd $\geq 1$. One thus sees that the ordinary Sachs-Wolfe contribution to the large multipoles is identical for all spherical spaces, including $\mathcal{S}^{3}$, in agreement with the expectation that the cosmic topology is most clearly seen at large scales.

The above discussion showed how the topology of the Universe influences via the vibrational modes the CMB anisotropy at large scales. The whole story is, however, 
more subtle, since an additional $l$-dependence comes in eq. (17) from the radial functions $R_{\beta l}\left(\tau_{\mathrm{SLS}}\right)$, and furthermore, there is an important dependence of the multipoles on the curvature radius respectively on $\Omega_{\text {tot }}$ which is determined by both the time evolution via $g_{\beta}\left(\eta_{\mathrm{SLS}}\right)$ and the radial functions. The interplay of all these effects is responsible for the rather complicated structure displayed by the numerical computations to be illustrated below.
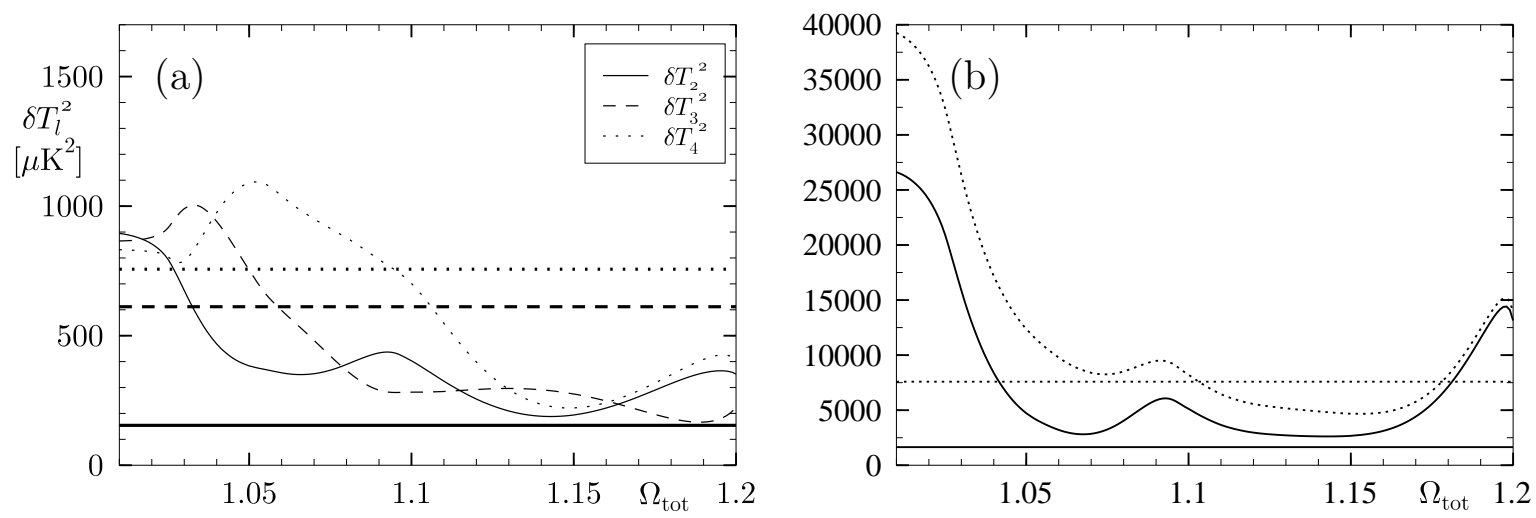

Figure 1. The binary tetrahedral group $T^{\star}$. Panel (a) shows the $\Omega_{\text {tot }}$ dependence of the mean value of the first three angular power moments $\delta T_{l}^{2}$. The horizontal lines indicate the corresponding WMAP values for $\delta T_{l}^{2}$. Panel (b) displays the $S(\rho)$ statistics $\left(\rho=60^{\circ}\right.$ full curve and $\rho=20^{\circ}$ dotted curve) in units of $\mu \mathrm{K}^{4}$ in dependence on $\Omega_{\text {tot }}$. The corresponding WMAP values are indicated as horizontal lines.
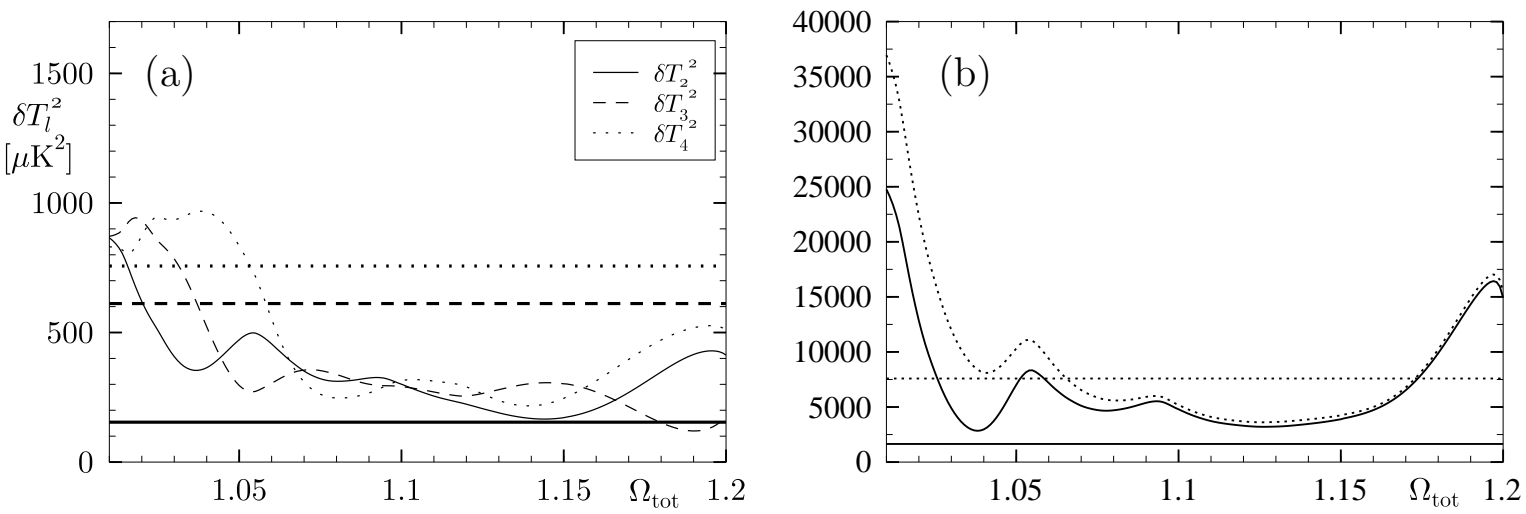

Figure 2. The same as in figure 1 for the binary octahedral group $O^{\star}$.

Let us now discuss the results for the different spherical space forms. In all these computations the CMB anisotropy is obtained using the complete Sachs-Wolfe formula $\left(\tau(\eta):=\eta_{0}-\eta\right)$

$$
\frac{\delta T}{T}(\hat{n})=\sum_{\beta \geq 3}^{\prime} \sum_{i=1}^{r^{\mathcal{M}}(\beta)}\left[\left(\Phi_{\beta}^{i}(\eta)+\frac{\delta_{\gamma, \beta}^{i}(\eta)}{4}+\frac{a(\eta) V_{\gamma, \beta}^{i}(\eta)}{E_{\beta}} \frac{\partial}{\partial \tau}\right) \Psi_{\beta}^{\mathcal{M}, i}(\tau(\eta), \theta, \phi)\right]_{\eta=\eta_{\mathrm{SLS}}}
$$



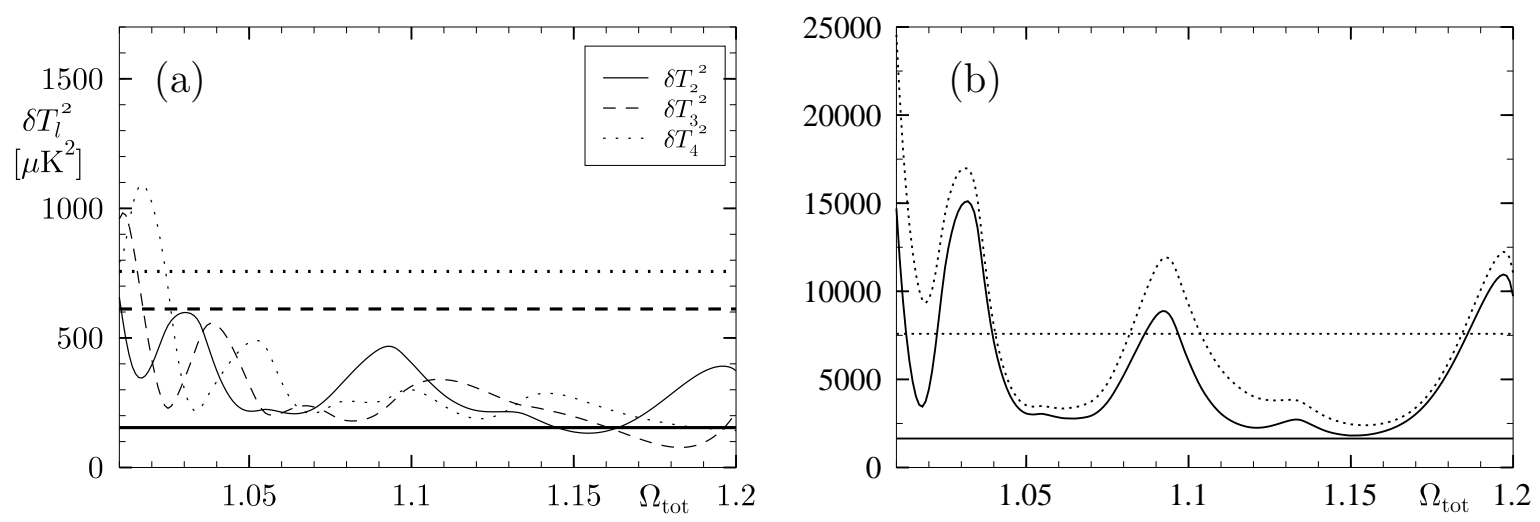

Figure 3. The same as in figure 1 for the binary icosahedral group $I^{\star}$.
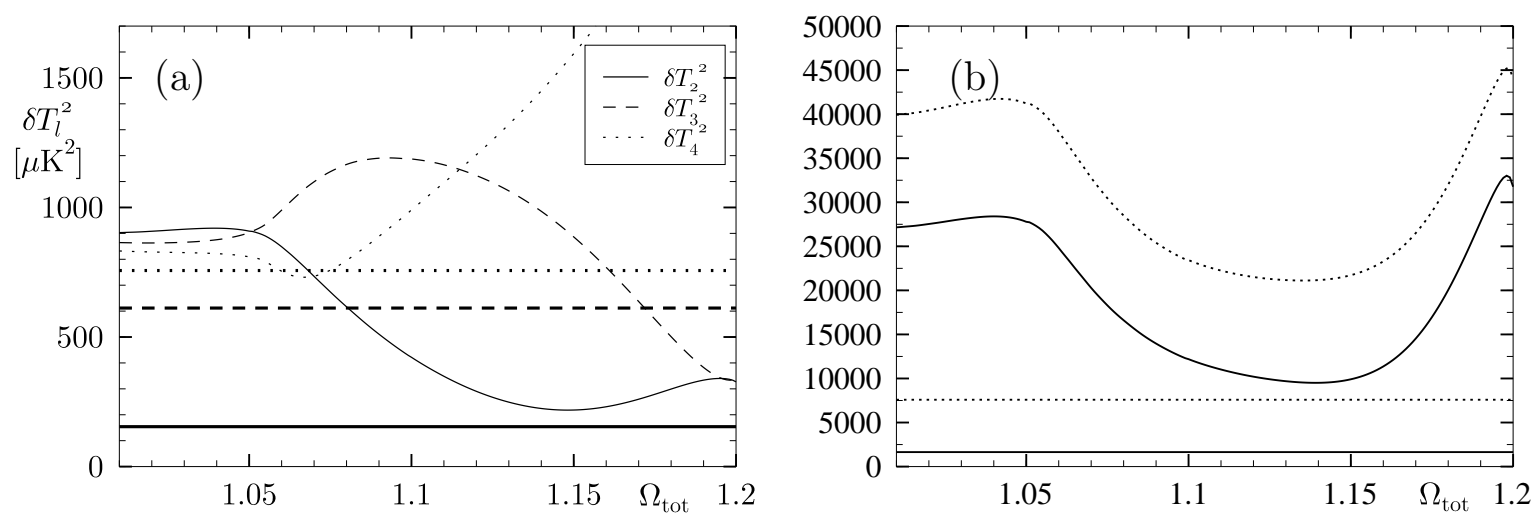

Figure 4. The same as in figure 1 for the binary dihedral group $D_{8}^{\star}$, i. e. $m=2$.
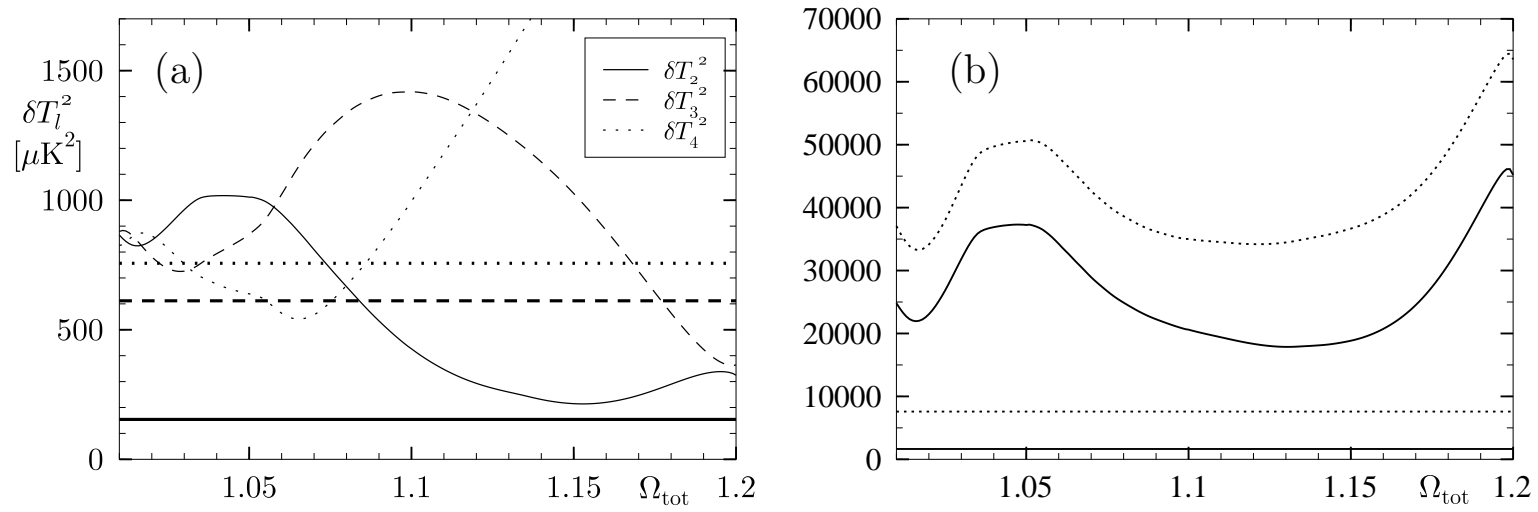

Figure 5. The same as in figure 1 for the binary dihedral group $D_{20}^{\star}$, i. e. $m=5$.

$$
+2 \sum_{\beta \geq 3}^{\prime} \sum_{i=1}^{r^{\mathcal{M}}(\beta)} \int_{\eta_{\mathrm{SLS}}}^{\eta_{0}} d \eta \frac{\partial \Phi_{\beta}^{i}(\eta)}{\partial \eta} \Psi_{\beta}^{\mathcal{M}, i}(\tau(\eta), \theta, \phi)
$$

The details of the computation of the quantities needed in (24) are described in [12]. The first two terms in (24) are the ordinary Sachs-Wolfe contribution (9) discussed 

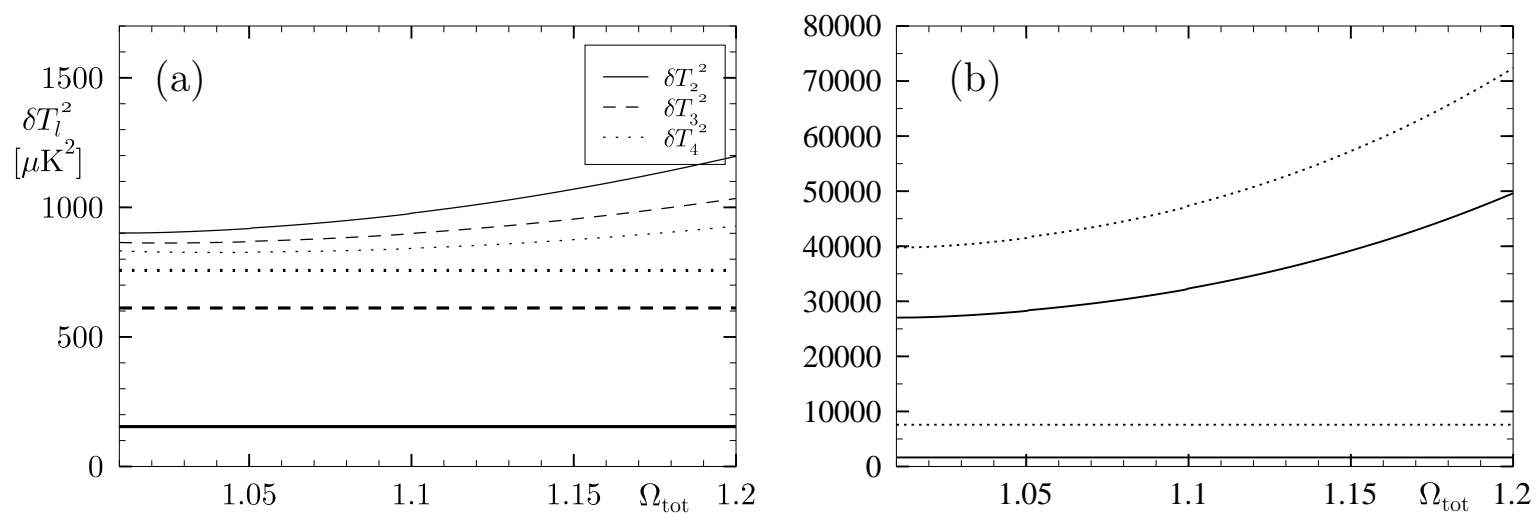

Figure 6. The same as in figure 1 for the cyclic group $Z_{1}$, i. e. for the space $\mathcal{S}^{3}$.
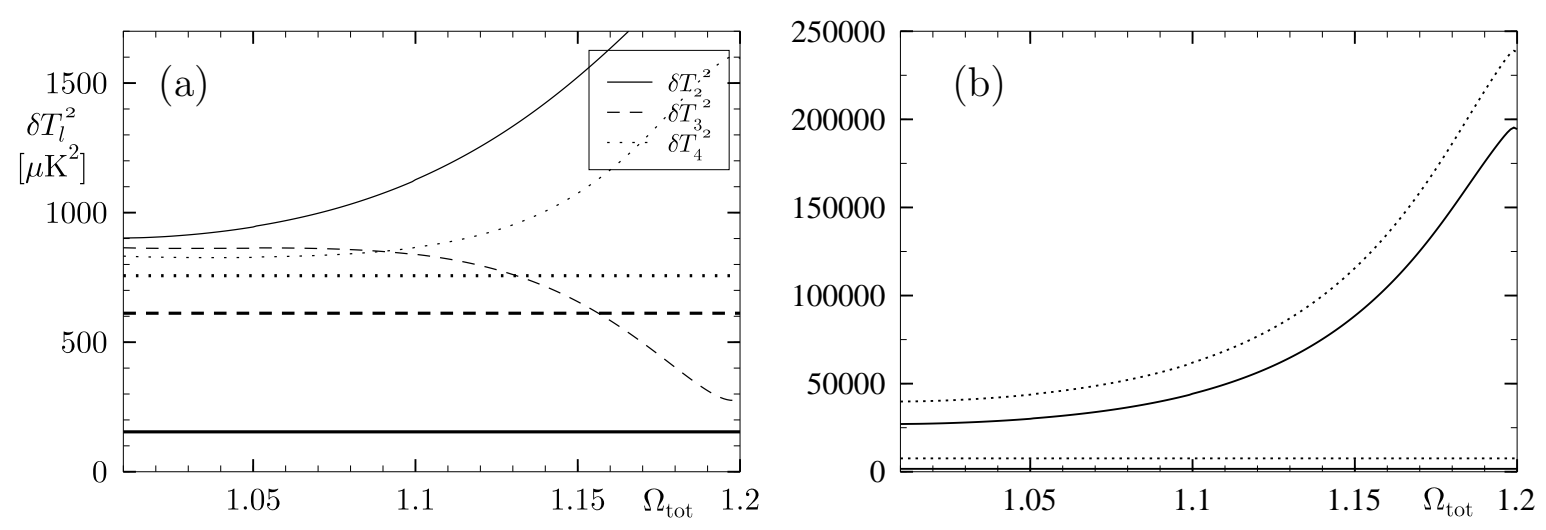

Figure 7. The same as in figure $\square$ for the cyclic group $Z_{2}$, i. e. for the projective space $\mathcal{P}^{3}$.
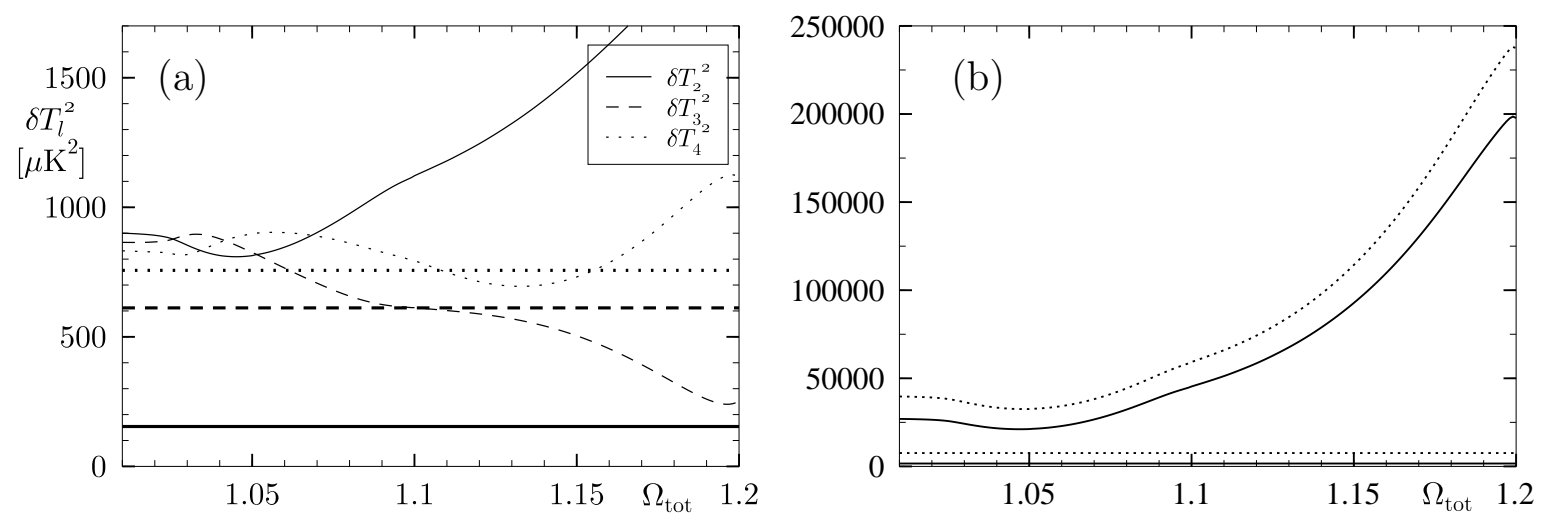

Figure 8. The same as in figure 1 for the cyclic group $Z_{6}$.

above. The next term involving the spatial covariant divergence of the velocity field is the Doppler contribution. The integral over the photon path yields the integrated Sachs-Wolfe contribution. The $\beta_{\max }$ cut-off is chosen sufficiently high in order to obtain enough multipoles which then enables us to normalise the $\delta T_{l}^{2}$ spectrum in the range 
$l=20$ to 45 according to the WMAP first-year data [10]. In our computations we use $\beta_{\max }=3001$ for $\Omega_{\mathrm{tot}} \leq 1.05, \beta_{\max }=2001$ for $1.05<\Omega_{\mathrm{tot}} \leq 1.1$, and $\beta_{\max }=1501$ for $1.1<\Omega_{\text {tot }} \leq 1.2$. In the following we fix the cosmological parameters as $\Omega_{\text {bar }}=0.046$, $\Omega_{\text {mat }}=0.28$, and $h=70$, in agreement with the WMAP data. The free parameter is $\Omega_{\Lambda}=\Omega_{\text {tot }}-\Omega_{\text {mat }}-\Omega_{\text {rad }}$, i. e. the density corresponding to the cosmological constant. Although $\Omega_{\Lambda}$ is varied, we show in the following figures $\Omega_{\text {tot }}$ on the abscissa.

In figures 11 to 8 we show the dependence of the large scale CMB anisotropy on $\Omega_{\text {tot }}$ for the binary tetrahedral space, the binary octahedral space, the dodecahedral space, two binary dihedral spaces and three spaces belonging to cyclic groups. In panel (a) the expectation values of the angular power spectra $\delta T_{l}^{2}$ are shown for the first three multipole moments $\delta T_{2}^{2}$ (solid curve), $\delta T_{3}^{2}$ (dashed curve) and $\delta T_{4}^{2}$ (dotted curve). (Panel (b) in figures 1 to 8 will be discussed below.) The corresponding values measured by the WMAP team are indicated as straight horizontal lines. With respect to the large scale anisotropy, the best values for $\Omega_{\text {tot }}$ are obtained by choosing those values which yield the best agreement with the data. For example, consider the binary tetrahedral space shown in figure 1. Since the density $\Omega_{\text {tot }}$ should be as close to one as possible, one chooses the first minimum of $\delta T_{2}^{2}$, i. e. the range $\Omega_{\text {tot }}=1.06 \ldots 1.07$. In this range the value of $\delta T_{3}^{2}$ is also near to the observed one. One should note that these values are only the expectation values such that for a given realization one has also to take into account the cosmic variance. However, as the simulations for such realizations show, the probability for a given spherical space increases significantly when the expectation values are already near to the observed values. In this way one gets for the binary octahedral space (figure 2) the range $\Omega_{\text {tot }}=1.03 \ldots 1.04$ and for the dodecahedron (figure 3) $\Omega_{\text {tot }}=1.015 \ldots 1.02$.

For none of the considered binary dihedral spaces is a comparably good agreement found. We have computed the CMB anisotropy for the binary dihedral groups $D_{4 m}^{\star}$ with $m=2,3,4,5,10,20$, and 30. In figures 4 and 5 we show as two typical examples the cases $m=2$ and $m=5$, respectively. The first multipole moment $\delta T_{2}^{2}$ decreases for all considered groups $D_{4 m}^{\star}$ only for unrealistically high values of $\Omega_{\text {tot }}>1$.1. In this parameter range the values for $\delta T_{4}^{2}$ are very large. Thus a binary dihedral topology seems to be very unprobable as a possibility for our Universe.

The cyclic groups $Z_{m}$ are also much worse compared to the binary tetrahedral space, the binary octahedral space, or the dodecahedron. We have computed all groups $Z_{m}$ with $m \leq 20$ and a lot of examples up to $m=500$. As three examples we present in figures 6 to 8 the models for $m=1,2$, and 6 , respectively. The group $Z_{1}$ has only the identity as a group element and thus leads to the usual spherical space $\mathcal{S}^{3}$. One observes the known difficulty of the concordance model, i. e. too large values for the two lowest multipole moments. The next group shown is $Z_{2}$ leading to the projective space $\mathcal{P}^{3}$, also known as elliptic space, which has historically played a special role as an example of an alternative to the spherical space $\mathcal{S}^{3}$. For $\Omega_{\text {tot }}<1.1$ the first multipole moments behave very similar to the former case with $m=1$ such that this topology is not a better match than the concordance model. Models with larger groups $Z_{m}$ lead to 
a quadrupole moment which increases with increasing $\Omega_{\text {tot }}$, in general. As an example, figure 8 displays the result for $Z_{6}$. The quadrupole moment $\delta T_{2}^{2}$ shows a very small minimum around $\Omega_{\text {tot }}=1.05$ and increases for higher values of $\Omega_{\text {tot }}$. Although there are minima in many cyclic models, they never suppress the power of $\delta T_{2}^{2}$ even to the level of the observed value for $\delta T_{4}^{2}$, see figure 8. The fact that lens spaces do not fit well the CMB anisotropy is already discussed in [30] where this behaviour is ascribed to the non well-proportioned fundamental cells.

Therefore, from all spherical spaces, only the binary tetrahedral space, the binary octahedral space, and the dodecahedron display for the given parameter ranges the observed suppression in the large anisotropy power.

Up to now, we have only discussed the angular power spectrum $\delta T_{l}^{2}$. Let us now come to the temperature two-point correlation function $C(\vartheta)$, which is defined as $C(\vartheta):=\left\langle\delta T(\hat{n}) \delta T\left(\hat{n}^{\prime}\right)\right\rangle$ with $\hat{n} \cdot \hat{n}^{\prime}=\cos \vartheta$. It can be computed from the multipole moments (14) under the assumption of statistical isotropy as

$$
C(\vartheta) \simeq \frac{1}{4 \pi} \sum_{l=2}^{\infty}(2 l+1) C_{l} P_{l}(\cos \vartheta)
$$

This quantity is well suited in order to measure the large scale power as emphasised in [8] where the observations are compared with the theoretical models. A comparison of $C(\vartheta)$ observed by WMAP with the concordance model can also be found in figure 1 of reference [14. (In [14] we have derived an analytic expression for $C^{\mathrm{SW}}(\vartheta)$, i. e. for the SW contribution in the case of the dodecahedron, which after multiplication by $N / 120$ holds for all homogeneous spherical space forms.) The correlation function $C(\vartheta)$ displays a surprisingly low CMB anisotropy on large angular scales $\vartheta \geq \rho$, which can be quantified by the $S(\rho)$ statistic [10]

$$
S(\rho)=\int_{-1}^{\cos \rho}|C(\vartheta)|^{2} d \cos \vartheta
$$

which is discussed for the first-year WMAP data in [8] for $\rho=60^{\circ}$, and it is found that only $0.3 \%$ of the simulations based on the concordance model ri have lower values of $S\left(60^{\circ}\right)$ than the observed value $S\left(60^{\circ}\right)=1644$. The $S(\rho)$ statistic is shown for the above discussed spherical spaces in figures 1 to 8 in panel (b) for $\rho=60^{\circ}$ (solid curves) and $\rho=20^{\circ}$ (dotted curves). The corresponding WMAP values are indicated as straight horizontal lines. The inspection of these figures leads to the same result as the above discussion of the first three angular power moments $\delta T_{l}^{2}$. The binary tetrahedral space possesses a sufficiently low power in the range $\Omega_{\text {tot }}=1.06 \ldots 1.07$, the binary octahedral space in the range $\Omega_{\text {tot }}=1.03 \ldots 1.04$, and the dodecahedral space in the range $\Omega_{\text {tot }}=1.015 \ldots 1.02$. For the other spherical spaces no comparable agreement is found as can be seen in the case of the two binary dihedral spaces (figures 4(b) and [5(b)) and the three cyclic groups (figures 6(b) to 8(b)).

Now, we would like to demonstrate the strong influence of the radial function $R_{\beta l}^{2}(\tau)$ on the suppression of power in the case of the quadrupole moment $l=2$ already discussed above. The quadrupole suppression gets stronger, the higher the value of the 

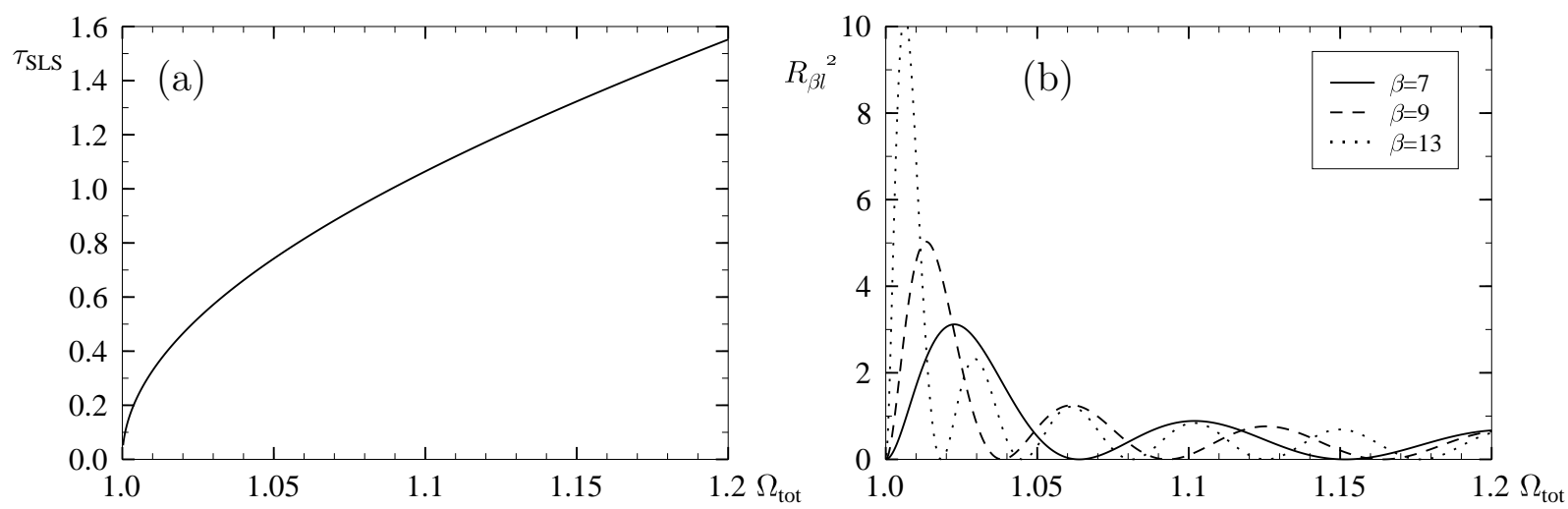

Figure 9. Panel (a) shows the dependence of the conformal distance $\tau_{\text {SLS }}$ to the surface of last scattering on the density $\Omega_{\text {tot }}$. Panel (b) shows the square of the radial function $R_{\beta l}^{2}(\tau)$ for the first eigenvalue occurring in (24) for the quadrupole moment $l=2$ for the binary tetrahedral space $(\beta=7)$, the binary octahedral space $(\beta=9)$, and the dodecahedron $(\beta=13)$.

first contributing eigenvalue is, see equation (17). In addition, it is seen from equation (17) that the term belonging to the first eigenvalue $\beta$ is multiplied by $R_{\beta l}^{2}(\tau)$. Thus the contribution of the first eigenvalue is eliminated for those values of $\Omega_{\text {tot }}$ which belong to a value of $\tau_{\text {SLS }}$ at which $R_{\beta l}\left(\tau_{\text {SLS }}\right)$ is zero. The dependence of $\tau_{\text {SLS }}$ on the density $\Omega_{\text {tot }}$ is shown in figure 9(a) for our choice of cosmological parameters and is well described by $\tau_{\mathrm{SLS}}=3.32 \sqrt{\Omega_{\mathrm{tot}}-1}$ for $1<\Omega_{\mathrm{tot}}<1.1$. In figure 9 (b) the square of the radial function $R_{\beta l}^{2}(\tau)$ is shown for the first eigenvalue of the binary tetrahedral space $(\beta=7)$, the binary octahedral space $(\beta=9)$, and the dodecahedron $(\beta=13)$ for $l=2$. One observes that the quadrupole suppression due to the first zero of the radial function is maximal in the case of the binary tetrahedral space at $\Omega_{\text {tot }} \simeq 1.064$, at $\Omega_{\text {tot }} \simeq 1.038$ for the binary octahedral space, and at $\Omega_{\mathrm{tot}} \simeq 1.018$ for the dodecahedral space. This matches perfectly well to the previously found intervals on which these models show a strong anisotropy suppression. Thus, the radial function has an important influence on the suppression for a given spherical topology. Note that the zero of the radial function eliminates many eigenfunctions due to the high degeneracy, e. g. the first 13 eigenfunctions in the case of the dodecahedron. This does not happen in such a dramatic way in models with negative curvature, where one has no degeneracies at all, i. e. all eigenvalues have multiplicity one, in general. Then the radial function can only suppress a single eigenfunction and not a "cluster" of them.

The angular power spectrum $\delta T_{l}^{2}$ as well as the $S(\rho)$ statistic lead to the conclusion that there are three best candidates with respect to spherical spaces, i. e. the binary polyhedral spaces $\mathcal{S}^{3} / T^{\star}, \mathcal{S}^{3} / O^{\star}$, and $\mathcal{S}^{3} / I^{\star}$. In figures 10 to 12 we show the temperature fluctuation $\delta T / T$ in the Mollweide projection for these three topological spaces, where exactly those values of $\Omega_{\text {tot }}$ are used which lead to a strong suppression of large scale power. In these calculations we have used for the three spaces all modes below the wave number cut-off $\beta_{\max }=155,161$ and 185, respectively. 


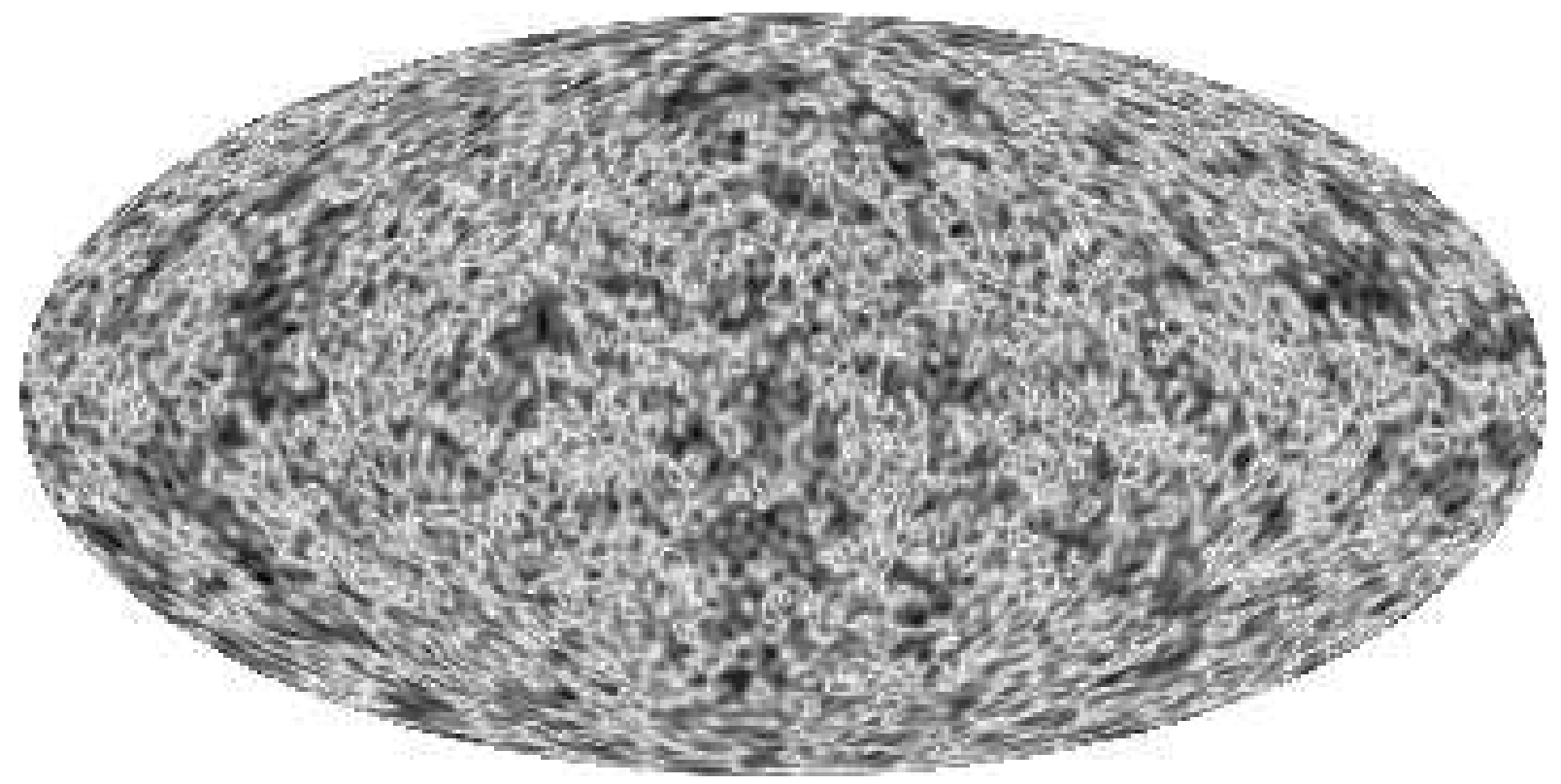

Figure 10. The temperature fluctuation $\delta T / T$ of one realization for the binary tetrahedral group $T^{\star}$ is shown $\left(\beta_{\max }=155\right)$. The cosmological parameters $\Omega_{\mathrm{tot}}=$ $1.065, \Omega_{\Lambda}=0.785$ and $h=70$ are used.

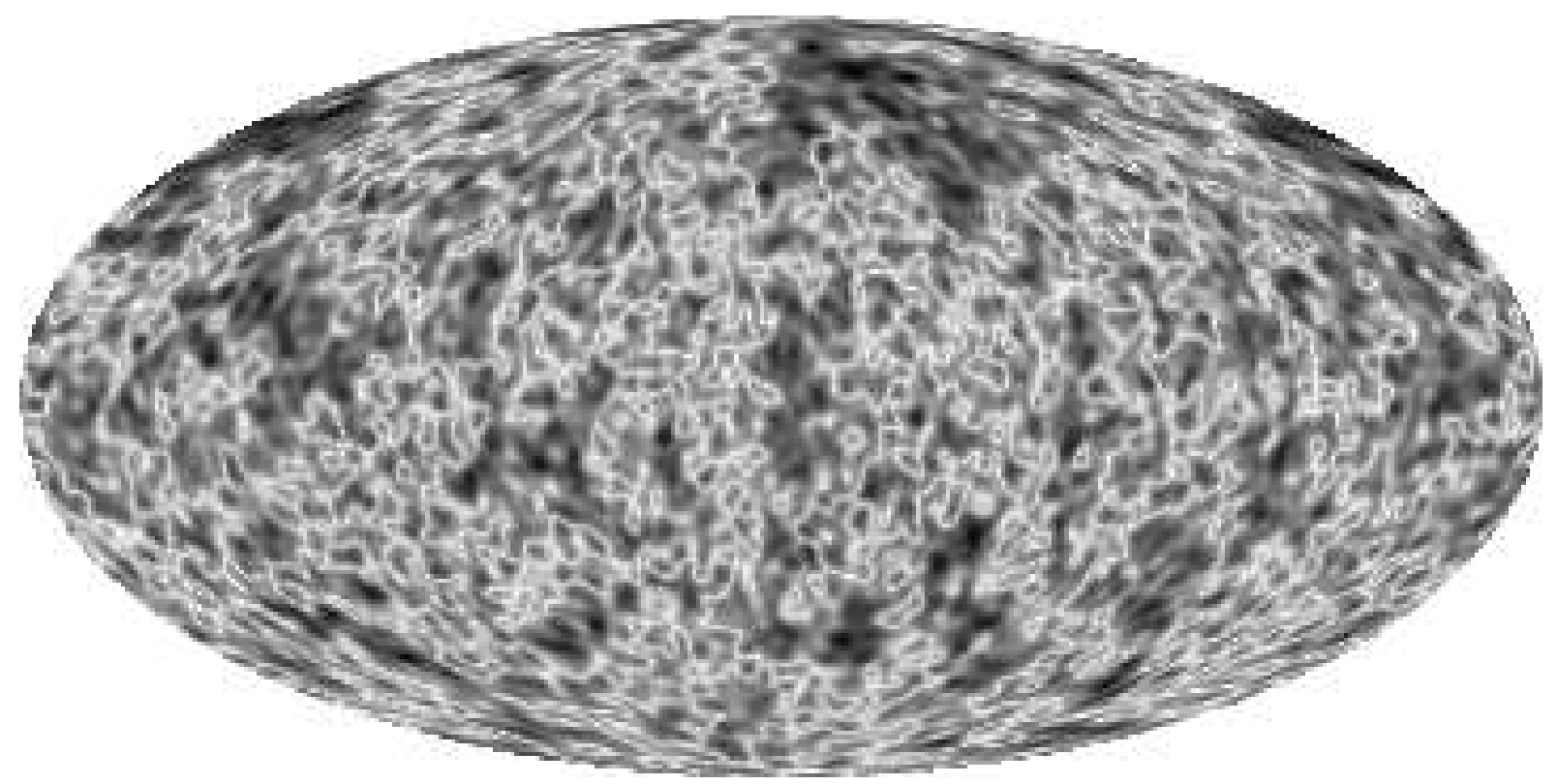

Figure 11. The temperature fluctuation $\delta T / T$ of one realization for the binary octahedral group $O^{\star}$ is shown $\left(\beta_{\max }=161\right)$. The cosmological parameters $\Omega_{\mathrm{tot}}=$ $1.038, \Omega_{\Lambda}=0.758$ and $h=70$ are used.

In figures 13 to 15 we show the angular power spectrum $\delta T_{l}^{2}$ for the binary polyhedral spaces $\mathcal{S}^{3} / T^{\star}, \mathcal{S}^{3} / O^{\star}$, and $\mathcal{S}^{3} / I^{\star}$, where the same cosmological parameters as in figures 10 to 12 are used. The $1 \sigma$ deviations are computed along the lines of [15. Since the distributions for the lowest multipole moments are asymmetric, i. e. not 


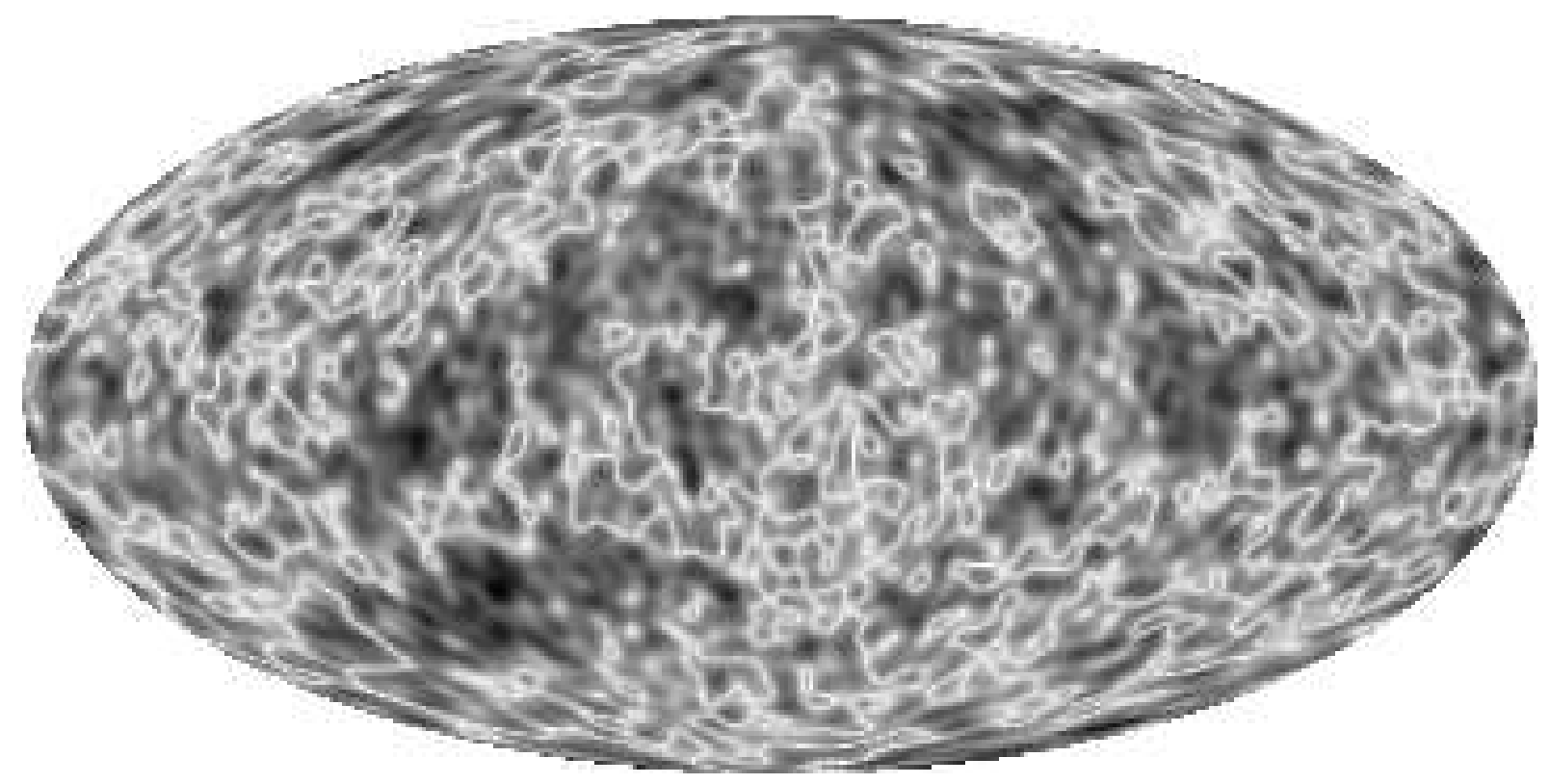

Figure 12. The temperature fluctuation $\delta T / T$ of one realization for the binary icosahedral group $I^{\star}$ is shown $\left(\beta_{\max }=185\right)$. The cosmological parameters $\Omega_{\text {tot }}=$ $1.018, \Omega_{\Lambda}=0.738$ and $h=70$ are used.

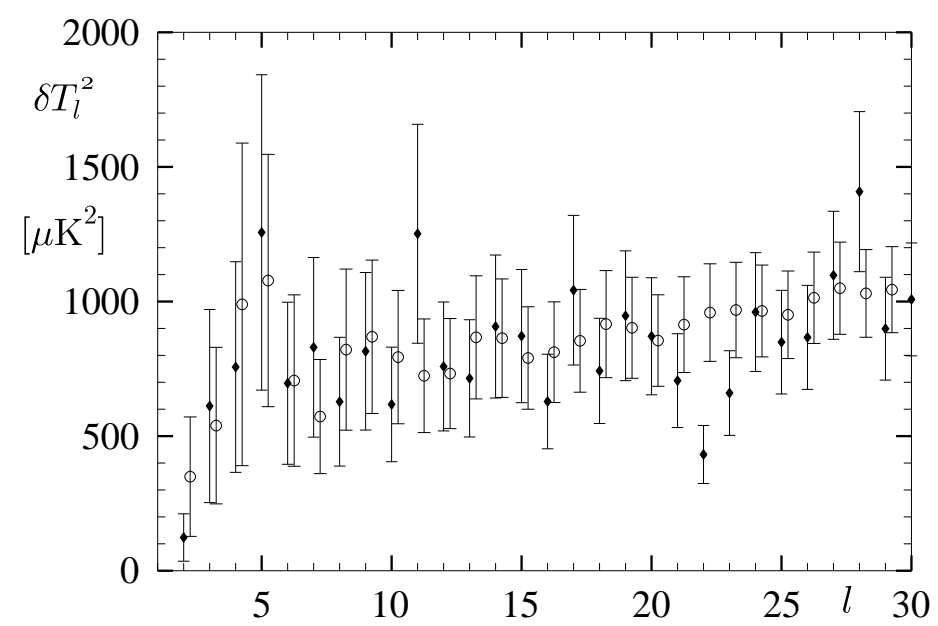

Figure 13. The angular power spectrum $\delta T_{l}^{2}$ is shown for the binary tetrahedral group $T^{\star}$ (open circles) using the same cosmological parameters as in figure [10] The angular power spectrum is shifted by $\Delta l=0.25$ in order to enable a comparison with the first-year WMAP data (full diamonds). The $1 \sigma$ errors are shown.

Gaussian, these error bars should only be considered as providing the order of magnitude of fluctuations in individual realizations. In order to facilitate a comparison with the WMAP data, shown as full diamonds together with their $1 \sigma$ errors not including the cosmic variance, the spectra of the binary polyhedral spaces are shifted by $\Delta l=0.25$. The angular power spectra $\delta T_{l}^{2}$ for these three binary polyhedral spaces are very similar such that one is faced with a topological degeneracy with respect to $\delta T_{l}^{2}$. All three 


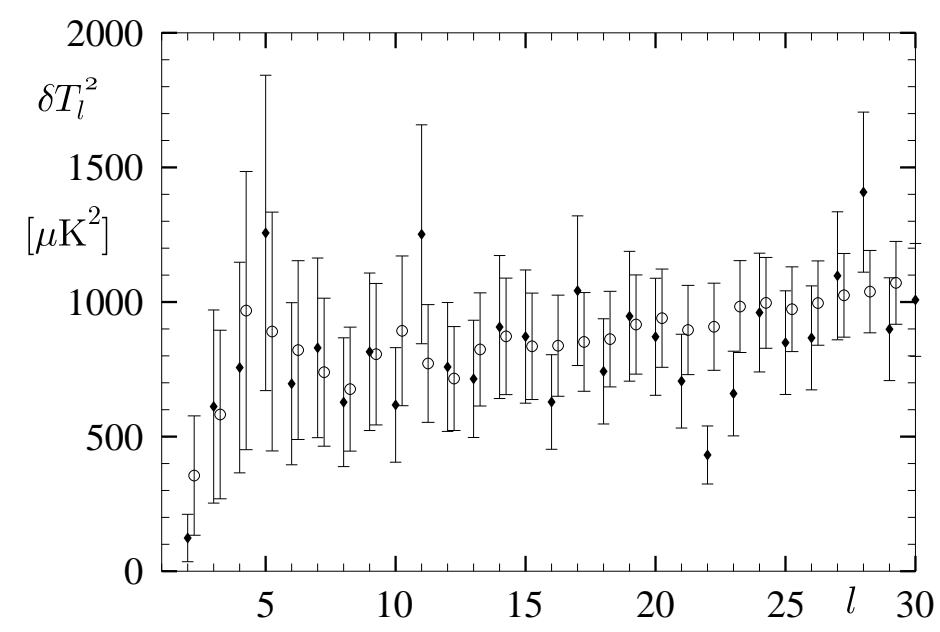

Figure 14. The angular power spectrum $\delta T_{l}^{2}$ is shown for the binary octahedral group $O^{\star}$ (open circles) using the same cosmological parameters as in figure 11]

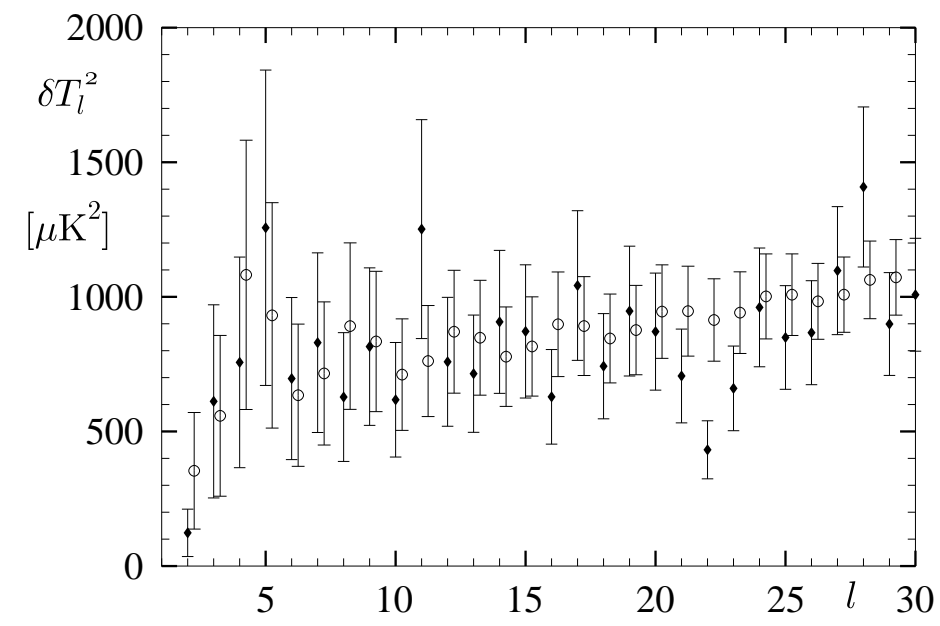

Figure 15. The angular power spectrum $\delta T_{l}^{2}$ is shown for the binary icosahedral group $I^{\star}$ (open circles) using the same cosmological parameters as in figure 12

spectra display a good agreement with the WMAP data.

\section{Conclusion}

In this paper we analyse the CMB anisotropy of homogeneous 3-spaces of constant positive curvature which are multi-connected and are given by the quotient of $\mathcal{S}^{3}$ by a group $\Gamma$ of covering transformations, i. e. $\mathcal{M}=\mathcal{S}^{3} / \Gamma$. The motivation is provided by the surprisingly low power in the CMB anisotropy at the largest scales as measured by COBE and WMAP and the fact that the mean value of $\Omega_{\text {tot }}$ reported by WMAP is 1.020 which hints to a positively curved Universe. In order to explain this low power, one could modify the primordial power spectrum $P_{\Phi}(\beta)$, e. g. by carefully choosing the inflationary scalar potential, or by resorting to multi-connected space forms which give 
a low CMB anisotropy at the largest scales due to missing modes compared to the simply-connected $\mathcal{S}^{3}$, in general.

We study all types of homogeneous multi-connected spherical space forms and find no agreement for the cyclic groups $Z_{m}$ which show an enhanced power at the largest scales despite their small volumes. Also the binary dihedral groups $D_{4 m}^{\star}$ do not lead to models with a suppression significantly stronger than the simply-connected $\mathcal{S}^{3}$ universe. Thus these models do not seem to provide viable space forms as a model for our Universe. This contrasts to the remaining three space forms, the binary tetrahedral, the binary octahedral as well as the dodecahedral space forms which show a sufficiently strong suppression of large scale power compared to the simply-connected $\mathcal{S}^{3}$ universe. The binary tetrahedral space requires a density $\Omega_{\text {tot }}$ in the range $1.06 \ldots 1.07$. Since the WMAP team reported $\Omega_{\text {tot }}=1.02 \pm 0.02$ this model is probably in conflict with the observations. For the two remaining models the density $\Omega_{\text {tot }}$ should be in the

range $\Omega_{\mathrm{tot}}=1.03 \ldots 1.04$ for the binary octahedral space, and $\Omega_{\mathrm{tot}}=1.015 \ldots 1.02$ for the dodecahedral space. These values are compatible with the current observations. Furthermore, we would like to remark that the binary octahedral space displays a slightly stronger suppression of power than the dodecahedral space, as a comparison of figures 2 and figures 3 reveals.

A unique signal for a particular topology is provided by the so-called circles-in-thesky-signature proposed in 31. Along two circles on the sky which are mapped onto each other by the group $\Gamma$, the ordinary Sachs-Wolfe effect produces the same temperature signal. If there would be no Doppler and integrated Sachs-Wolfe contribution, see equation (24), which disturb this signal, one would expect a clear sign for a given topology if present. In [14] we study the influence of the latter two contributions on the circles-in-the-sky-signature and find that the degradation of the signal is strong enough such that the topology signal can be swamped. Therefore, the fact that in 32 no circles are found in the WMAP sky maps, does not necessarily exclude the binary octahedral or the dodecahedral space as viable models for our Universe. In a forthcoming paper we will study the circles-in-the-sky for the three best multi-connected spherical space forms, and in particular shall discuss whether a combined circle search on all circles simultaneously of a given topology can overcome the degradations.

\section{Acknowledgment}

One of us (F.S.) would like to thank the Theoretical Physics Division of CERN for hospitality.

\section{References}

[1] K. Schwarzschild, Vierteljahrsschrift der Astron. Gesellschaft 35, 337 (1900).

[2] A. Einstein, Sitzungsber. Preuß. Akad. Wiss. , 142 (1917).

[3] A. Friedmann, Zeitschrift f. Physik 10, 377 (1922).

[4] A. Friedmann, Zeitschrift f. Physik 21, 326 (1924). 
[5] G. Lemaitre, The primaeval atom hypothesis and the problem of the clusters of galaxies, in $L a$ Structure et l' Évolution de l' Univers, pp. 1-31, Coudenberg, Bruxelles, 1958, Rapports et discussions du $11^{\circ}$ Conseil de physique, Institut International de Physique Solvay, R. Stoops (ed.).

[6] O. Heckmann and E. Schücking, in: Handbuch der Physik, Vol. 53: "Newtonsche und Einsteinsche Kosmologie", 489-519 (Springer, Berlin, 1959), S. Flügge (ed.).

[7] G. F. R. Ellis, Gen. Rel. Grav. 2, 7 (1971).

[8] D. N. Spergel et al., Astrophys. J. Supp. 148, 175 (2003), astro-ph/0302209.

[9] G. Hinshaw et al., Astrophys. J. Lett. 464, L25 (1996).

[10] C. L. Bennett et al., Astrophys. J. Supp. 148, 1 (2003), astro-ph/0302207.

[11] J. Luminet, J. R. Weeks, A. Riazuelo, R. Lehoucq, and J. Uzan, Nature 425, 593 (2003).

[12] R. Aurich, S. Lustig, F. Steiner, and H. Then, Class. Quantum Grav. 21, 4901 (2004), astro$\mathrm{ph} / 0403597$.

[13] R. Aurich, S. Lustig, F. Steiner, and H. Then, Phys. Rev. Lett. 94, 021301 (2005), astro$\mathrm{ph} / 0412407$.

[14] R. Aurich, S. Lustig, and F. Steiner, Class. Quantum Grav. 22 (2005), astro-ph/0412569.

[15] J. Gundermann, astro-ph/0503014 (2005).

[16] W. Threlfall and H. Seifert, Math. Annalen 104, 1 (1930).

[17] W. Threlfall and H. Seifert, Math. Annalen 107, 543 (1932).

[18] J. A. Wolf, Spaces of constant curvature (Publish or Perish Boston, Mass., 1974), (Third edition).

[19] W. P. Thurston, Three-dimensional geometry and topology (Princeton Univ. Press, Princeton, NJ, 1997).

[20] A. Ikeda, Kodai Math. J. 18, 57 (1995).

[21] J. Weeks, math.SP/0502566 (2005).

[22] E. Schrödinger, Commentationes Pontificiae Academiae Scientiarium 2, 321 (1938).

[23] E. Schrödinger, Physica 6, 899 (1939).

[24] E. Schrödinger, Proc. Roy. Irish Academy 46A, 9 (1940).

[25] E. Schrödinger, Proc. Roy. Irish Academy 46A, 25 (1940).

[26] E. R. Harrison, Reviews of Modern Physics 39, 862 (1967).

[27] L. F. Abbott and R. K. Schaefer, Astrophys. J. 308, 546 (1986).

[28] E. Gausmann, R. Lehoucq, J. Luminet, J.-P. Uzan, and J. Weeks, Class. Quantum Grav. 18, 5155 (2001).

[29] J. M. Bardeen, Phys. Rev. D 22, 1882 (1980).

[30] J.-P. Uzan, A. Riazuelo, R. Lehoucq, and J. Weeks, Phys. Rev. D 69, 043003 (2004), astro$\mathrm{ph} / 0303580$.

[31] N. J. Cornish, D. N. Spergel, and G. D. Starkman, Class. Quantum Grav. 15, 2657 (1998).

[32] N. J. Cornish, D. N. Spergel, G. D. Starkman, and E. Komatsu, Phys. Rev. Lett. 92, 201302 (2004), astro-ph/0310233. 